\title{
A Revised Approach for the Life Prediction of Metal Materials Fabricated by Additive Manufacturing
}

\author{
Haiming HONG*, Jiaying WANG**, Peng LI*** \\ *Comprehensive Strength Department, Shenyang Aircraft Design Research Institute, Shenyang 110035, China, \\ E-mail: H_M_Hong@163.com \\ **Comprehensive Strength Department, Shenyang Aircraft Design Research Institute, Shenyang 110035, China, \\ E-mail: JY_Wang@163.com \\ ***School of Aeronautics Science and Engineering, BeiHang University, Beijing 100191, China, \\ E-mail:lpeng210@sina.com
}

crossref http://dx.doi.org/10.5755/j02.mech.28216

\section{Introduction}

As is well known, additive manufacturing (AM) technology is a new manufacturing technology, which has more advantages than the traditional manufacturing process in the efficient use of resources and the diversity of design [1]. In recent years, more and more metal components used in mechanical engineering industry are fabricated by $\mathrm{AM}$ techniques [2], [3]. In the engineering applications, many AM metal materials often bears the complicated fatigue loads, and thus the fatigue failure of AM metal materials becomes a very common phenomenon. Therefore, it is of great significance to propose an effective approach for predicting the fatigue life of AM metal materials.

Literature review shows that the fatigue performance of AM alloy components is mainly studied by experiments, and there is not much research on the theoretical prediction of fatigue life. Zhang [4], [6] et al. studied the effects of AM processing parameters on fatigue and fracture characteristics of $316 \mathrm{~L}$ stainless steel, and proposed a model to predict fatigue performance. Carneiro [7] carried out analysis on the cyclic response and fatigue performances for AM 17-4 PH stainless steel, and it was found that the strain-life fatigue curves showed an important key point between low cycle fatigue and high cycle fatigue. Furthermore, the defects and porosities by AM process could greatly affect the fatigue properties, and reduce the fatigue performances of the AM metal materials. Bagehorn [8] studied the application of mechanical surface finishing processes for roughness reduction and fatigue improvement of AM Ti-6Al-4V. It was found that the AM metallic parts showed a good surface roughness and an irreproducible and inhomogeneous surface morphology after the manufacturing process. Pegues [9] investigated the influence of surface roughness on the fatigue performances of AM Ti6Al4V, and it was found that fatigue performance is more related with the part diameter than that with the surface area. Sheridan [10] focused on the $\mathrm{AM}$ process parameters and porosity production for the AM alloy 718. And the fatigue life prediction was also studied. The results showed that it was suitable to adopt the component density as a metric in order to acquire the average pore size, however, it was necessary to conduct the data correlating two quantities first.

At present, the methods to study fatigue problems are mostly based on experiments and empirical formulas of statistics. However, this method relies on the fatigue test of a large number of components [11], which could consume a lot of manpower, material resources and financial resources. Therefore, in order to efficiently estimate the fatigue life of components, research on fatigue damage accumulation theory has been carried out, and various fatigue damage accumulation theories have been proposed [12] - [14]. As well known, continuum damage mechanics (CDM) is a branch of solid mechanics, which develops in recent decades. By introducing damage variables, CDM studies the weakening of mechanical performance for structural materials under fatigue loadings, and the final failure mechanism [15]. A lot of studies [16] - [19] are conducted to predict the fatigue damage behaviour of metal components in the engineering applications. The main advantage of CDM is that the mathematical form is relatively simple and the physical meaning is relatively clear. Currently, the fatigue accumulated theory based on CDM is an efficient approach for the fatigue life prediction of metal components.

In this paper, for the purpose of computing the fatigue life of AM alloy materials, we develop a fatigue damage model considering the AM effects. The AM parameters include laser power, scanning speed, laser spacing and powder layer thickness, which can be expressed by the volume energy density. First, the new fatigue dam-age evolution model is derived, and the calibration meth-od of material parameters is presented. After that, the numerical algorithm is presented for the life prediction. Finally, the fatigue lives of the AM alloy members manufactured by AM process are predicted, and the numerical results and the new theoretical model are verified by the experimental data.

\section{Theoretical model}

\subsection{Damage coupled elastoplastic model}

Within the framework of CDM theory [20], some basic concepts are proposed. For isotropic materials, the degradation of mechanical properties is usually characterized by the degradation of elastic modulus under cyclic loading. The deterioration of material properties appears as the decrease of material stiffness, which can be ex-pressed by damage variable $D$, as shown below:

$$
D=\frac{E-E_{D}}{E},
$$

where: $E$ is the Young's Modulus without damage and $E_{D}$ is the Young's Modulus with damage. As $E_{D}$ ranges from 0 to 


\section{$E, D$ ranges from 0 and 1 .}

For the materials without damage, the elastoplastic constitutive model could be expressed as:

$$
\varepsilon_{i j}^{e}=\frac{1+v}{E} \sigma_{i j}-\frac{v}{E} \sigma_{k k} \delta_{i j}
$$

where: $\sigma_{i j}$ is the stress tensor and $\varepsilon_{i j}^{e}$ is the elastic strain tensor; $v$ is poisson's ratio and $\delta_{i j}$ is Kronecker symbol. According to the concept of effective stress, we obtain:

$$
\tilde{\sigma}_{i j}=\frac{\sigma_{i j}}{1-D} .
$$

For the damaged materials, the linear elastic constitutive model can be obtained according to the equivalent strain hypothesis. By replacing the stress tensor $\sigma_{i j}$ in the above model with the effective stress tensor $\tilde{\sigma}_{i j}$ :

$$
\varepsilon_{i j}^{e}=\frac{1+v}{E}\left(\frac{\sigma_{i j}}{1-D}\right)-\frac{v}{E}\left(\frac{\sigma_{k k} \delta_{i j}}{1-D}\right) .
$$

The plastic constitutive model considers isotropic hardening and kinematic hardening, which can describe the plastic behaviour of metal materials. In order to describe the plastic material with hardening and damage, the damage mechanics equivalent stress is introduced. It is known that the total strain $\varepsilon_{i j}$ is generally decomposed into the elastic component $\varepsilon_{i j}^{e}$ and plastic component $\varepsilon_{i j}^{p}$ :

$$
\varepsilon_{i j}=\varepsilon_{i j}^{e}+\varepsilon_{i j}^{p}
$$

where: $s_{i j}$ is the stress deviator part; $X_{i j}$ is the back stress component and $Q$ is the radius of the isotropic hardening yield surface. The evolution of $Q$ is defined as:

$$
\dot{Q}=\dot{\lambda} b\left(Q_{\infty}-Q\right)
$$

where: $b$ and $Q_{\infty}$ are the material constants; $\dot{\lambda}$ is the plastic multiplier in the form of rate, which is determined by the consistent condition of plastic flow. Here, the nonlinear hardening model is used to describe the hardening behaviour of materials:

$$
X_{i j}=\sum_{k=1}^{M} X_{i j}^{(k)}
$$

The evolution of plastic strain component can be expressed as:

$$
\begin{aligned}
& \dot{\varepsilon}_{i j}^{p}=\frac{3}{2} \frac{\dot{\lambda}}{1-D}\left(\frac{s_{i j}}{1-D}-X_{i j}\right) /\left(\frac{s_{i j}}{1-D}-X_{i j}\right)_{e q v}, \\
& \dot{p}=\sqrt{\frac{2}{3} \dot{\varepsilon}_{i j}^{p} \dot{\varepsilon}_{i j}^{p}}=\frac{\dot{\lambda}}{1-D},
\end{aligned}
$$

$$
\dot{X}_{i j}^{(k)}=\left(\frac{2}{3} C_{k} \dot{\varepsilon}_{i j}^{p}-\gamma_{k} X_{i j}^{(k)} \dot{p}\right)(1-D) .
$$

\subsection{Fatigue damage model}

Considering the different stress states of the material subjected to the fatigue loading, it is necessary to employ different damage evolution equations to describe the damage evolution laws of materials under different stress states.

For the condition of uniaxial cycle loading, based on the continuum damage mechanics, the fatigue damage model is shown as follows [21]:

$$
\frac{d D}{d N}=\left[1-(1-D)^{\beta+1}\right]^{\alpha}\left[\frac{\sigma_{a}}{m\left(1-n \sigma_{m}\right)(1-D)}\right]^{\beta},
$$

where: $\sigma_{a}$ and $\sigma_{m}$ are the stress amplitude and average stress, respectively. $\alpha, \beta, m$ and $n$ are the material constants.

However, in the real engineering problems, the stress and strain are often multiaxial for the components subjected to the cyclic loadings. In this case, the fatigue damage model is expressed as follows:

$$
\frac{d D}{d N}=\left[1-(1-D)^{\beta+1}\right]^{\alpha}\left[\frac{A_{I I}}{m\left(1-n \sigma_{H, m}\right)(1-D)}\right]^{\beta},
$$

where: $A_{I I}$ is the shear stress amplitude.

$$
A_{I I}=\frac{1}{2}\left[\frac{3}{2}\left(S_{i j, \max }-S_{i j, \min }\right) \cdot\left(S_{i j, \text { max }}-S_{i j, \min }\right)\right]^{1 / 2},
$$

in which $S_{i j, \max }$ and $S_{i j \text {,min }}$ represent the maximum and the minimum deviatoric stresses in a loading cycle. $\sigma_{H, m}$ is the mean stress:

$$
\sigma_{H, m}=\frac{1}{6}[\max (\operatorname{tr}(\sigma))+\min (\operatorname{tr}(\sigma))],
$$

with $\operatorname{tr}(\sigma)=\sigma_{11}+\sigma_{22}+\sigma_{33}$.

Obviously, it is known that in the traditional uniaxial and multiaxial fatigue damage models, there are no terms related to the AM parameters. Therefore, the two equations are not suitable for fatigue life prediction of AM materials. Furthermore, literature review has shown that the AM parameters (including laser power, scanning speed, laser spacing and layer thickness) are closely related to the porosity, manufacturing defects and microstructure of the material, which have a significant impact on the fatigue behaviour of AM materials. Here, considering the above four parameters can be expressed by the volume energy density, a new fatigue damage evolution equation is proposed.

First, under the uniaxial cyclic fatigue loadings, the fatigue damage model is shown below:

$$
\dot{D}=\frac{d D}{d N}=\left[1-(1-D)^{\beta+1}\right]^{\alpha}\left\{\frac{\sigma_{a}\left(1+k R_{E}\right)}{m\left(1-n \sigma_{m}\right)(1-D)}\right\}^{\beta} .
$$


Second, under the multiaxial cyclic fatigue loadings, the fatigue damage model is:

$$
\dot{D}=\frac{d D}{d N}=\left[1-(1-D)^{\beta+1}\right]^{\alpha} \cdot\left\{\frac{A_{I I}\left(1+k R_{E}\right)}{m\left(1-n \sigma_{H, m}\right)(1-D)}\right\}^{\beta},
$$

where: $R_{E}=E_{d} / E_{d 0}$ is the ratio of the volume energy density; $E_{d}$ is the volume capacity density and $E_{d 0}$ is the reference volume energy density. $k$ is the correction parameter, which is closely related with the AM process parameters. The AM metal components could have the minimum porosity with the reference volume energy density, and thus the correction parameter $k$ is set to be 0 when $E_{d}=E_{d 0}$.

Under the condition of the constant stress, the fatigue life $N_{F}$ could be computed with the integrals of Eqs. (15) and (16). For the case of uniaxial cycle loading:

$$
N_{f}=A\left\{\frac{\sigma_{a}\left(1+k R_{E}\right)}{\left(1-C \sigma_{m}\right)}\right\}^{-B} .
$$

For the case of multiaxial cycle loading:

$$
N_{f}=A\left\{\frac{A_{I I}\left(1+k R_{E}\right)}{\left(1-C \sigma_{H, m}\right)}\right\}^{-B},
$$

where: $\mathrm{A}=\frac{m^{\beta}}{(1-\alpha)(1+\beta)}, \quad \mathrm{B}=\beta, \quad \mathrm{C}=n$

\section{Calibrated method of parameter for AM metal mate- rials}

Two kinds of material parameters need to be calibrated in the elastoplastic damage analysis model, which are the static performance parameters used in the constitutive models and parameters in the fatigue damage models.

1. Parameter calibrated method in elastoplastic constitutive model.

First, the elastic modulus, yield stress and strength limit of the material can be calibrated directly by the uniaxial tensile static test. Second, the isotropic hardening parameters could be calculated by the relationship between the yield radius and cumulative plastic strain, which is based on the uniaxial tensile-compression cyclic test. Assuming that the material is subject to the complete kinetic hardening, the back stress equation employs three back stress components to describe the nonlinear hardening behavior of the material. The exponential expression of back stress is:

$$
X_{k}=\frac{C_{k}}{\gamma_{k}}\left(1-e^{-\gamma_{k} \varepsilon_{p}}\right)
$$

So the uniaxial stress-strain curve can be expressed as:

$$
\sigma=\sigma_{y 0}+\sum_{k=1}^{M} \frac{C_{k}}{\gamma_{k}}\left(1-e^{-\gamma_{k} \varepsilon_{p}}\right)
$$

2. Parameter calibrated method in fatigue damage model.
In the fatigue damage evolution equation, a total of 4 parameters need to be calibrated. According to the fatigue tests of standard smooth specimens under different stress ratios, the parameters can be calibrated by the least square method.

\section{Fatigue life prediction of AM metal materials}

\subsection{Numerical method}

In order to consider the coupling effects of fatigue damage model and elastoplastic constitutive model, it is necessary to write UMAT subroutines to implement the plastic stress-strain updating algorithm. In addition, the damage rate and cumulative damage could be computed by the UMAT subroutine at each material integration point after a cyclic loading. Because it takes a large amount of computer time to simulate each fatigue cycle, the jump-in-cycle method [22], [23] is employed, in which the damage is assumed to be constant in a cyclic block. The numerical calculation steps of fatigue life analysis are listed as follows.

1. Initialize all the parameters for the numerical simulation.

2. The ABAQUS software is used to compute the cyclic stress and strain under cyclic loadings. In the case of elastic damage, considering it is very time consuming in the cycling fatigue damage calculation, so the cyclic value of cyclic block is used. Here, it is important to note that the value of cyclic block is closely related to the convergence of numerical calculation. After that, the increment of fatigue damage and the total damage corresponding to the loading cycles are calculated.

3. If the damage accumulation at any element reaches 1 , it is assumed that the fatigue crack initiates at the element, and the fatigue life is obtained. If not, the material characteristics for each element are recalculated by the reduction of elastic modulus.

4. After that, the stress field and damage field are recalculated until the total damage accumulates to 1 at any element and the fatigue crack occurs.

\subsection{Predicted fatigue lives of AM metal materials}

\subsubsection{Fatigue life prediction for SS316L}

According to the proposed theoretical model and numerical method, the fatigue lives of AM SS316L are computed under different loading conditions. For SS316L specimens, according to the ASTM E466 standard, fatigue test [4] - [6] are carried out by the servo hydraulic MTS testing system. It is known that the force-controlled fatigue tests are conducted with the sinusoidal loading under the ambient conditions. A sample that did not fail after $10^{6}$ cycles was considered as a run-out. The maximum stress in the cyclic loading also changers in a relative wide range. The volume energy density ratio $R_{E}$ is in a wide range. The comparison between all the predicted results and the experimental data is shown in Fig. 1. It can be seen that the almost all the computed data are in accordance with the experimental fatigue lives, and only 12 sets exceed the three-error band. Furthermore, if the ratio $R_{E}$ is small, the fatigue life of AM SS316L could not be accurately computed by the proposed new theoretical model. Perhaps two items can explain for these data outside the three-error band. First, there is dispersion in the 
fatigue data of AM SS316L, especially when the ratio $R_{E}$ is smaller than the volume energy density ratio. Second, the porosity inside AM material becomes very high if the volume energy density ratio is too small, and there are a large number of defects inside AM components.

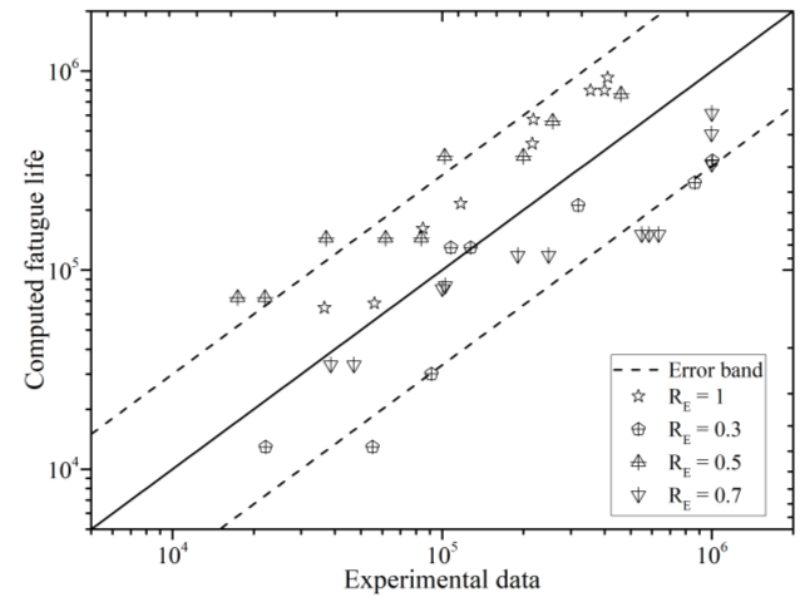

Fig. 1 Comparison between the predicted fatigue life and test results for AMSS316L alloy

Next, several important characteristics of fatigue behavior of SS316L material are discussed by the pro-posed fatigue damage model. Fig. 2 is shown for the changing curve of fatigue life with volume energy density ratio $R_{E}$ under the condition of different stress amplitudes and at the same stress ratio. First, we know that the fatigue life increases with the increase of $R_{E}$. Second, the smaller the stress amplitude is, the faster the growth trend of fatigue life is. And the larger the stress amplitude is, the less obvious of the increasing trend of fatigue life is. Third, when volume energy density ratio $R_{E}$ reaches 1.0 , the peak value of fatigue life is obtained, which means that the material has the very low porosity. Fig. 3 is shown for the changing curve of fatigue life with volume energy density ratio $R_{E}$ under different stress ratios with the same maximum stress. First, it is clear that the fatigue life increases rapidly with the increase of $R_{E}$. Second, for the same value of volume energy density ratio, the greater the stress ratio is, the longer the fatigue life is. Third, for different stress ratios, the fatigue life of AM $\mathrm{SS} 316 \mathrm{~L}$ could reach the maximum value if $R_{E}$ reaches 1.0 .

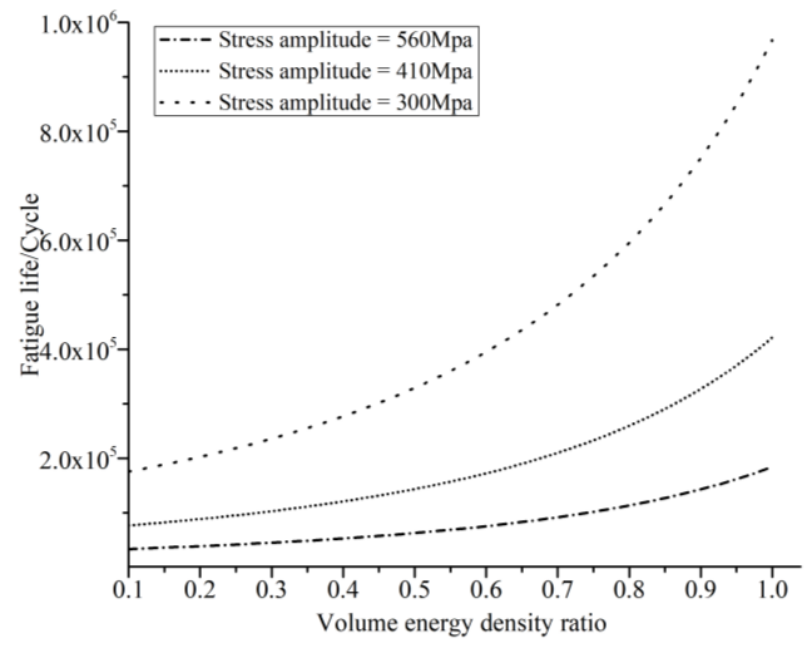

Fig. 2 Variation curve of AM SS316L fatigue life with volume energy density ratio under different stress amplitudes
Fig. 3 is shown for the changing curve of fatigue life with volume energy density ratio $R_{E}$ under different stress ratios at the maximum stress of $500 \mathrm{MPa}$. First, it is clear that the fatigue life increases rapidly with the increase of $R_{E}$. Second, for the same value of volume energy density ratio, the greater the stress ratio is, the longer the fatigue life is. Third, for different stress ratios, the fatigue life of AM $\mathrm{SS} 316 \mathrm{~L}$ could reach the maximum value if $R_{E}$ reaches 1.0.

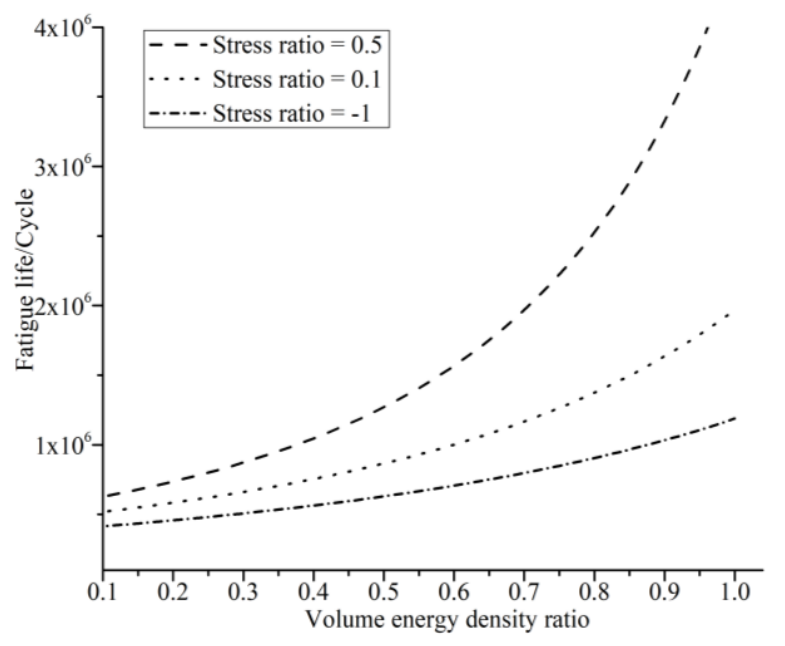

Fig. 3 Variation curve of AM SS316L fatigue life with volume energy density ratio under different stress ratios

\subsubsection{Fatigue life prediction for Ti6Al4V}

As well known, Ti6Al4V material is often employed in the field of mechanical engineering. At present, a large number of literatures have studied the fatigue properties of Ti6Al4V material. In this section, based on the proposed fatigue damage evolution equation, the fatigue lives of Ti6Al4V alloy are predicted and compared with the experimental results.

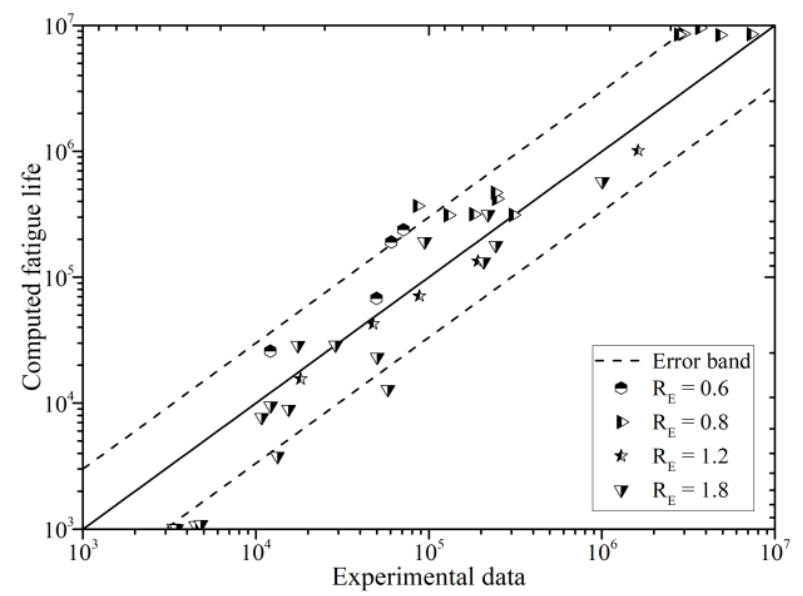

Fig. 4 Comparison between the predicted fatigue life and test results for AM Ti6Al4V alloy

In the fatigue test of Ti6Al4V alloy parts [24] [26], the stress ratio is different, and the maximum stress changes in a wide range. The computed results and the experimental data are given in Fig. 4. The results show that the computed fatigue lives are basically in accordance with the experimental data. Here, there are two possible reasons for these data out of the three-error band. On one hand, it is directly caused by the dispersion of fatigue data. On the other 
hand, when the value of volume energy density exceeds 1.0, which maybe meaning that the laser scanning speed is too fast, thus there could be some un-melted particles within AM alloys, and it has a great impact on the fatigue life. Therefore, the corresponding predicted fatigue lives is not accurate.

Next, the two important characteristics of fatigue behavior of Ti6Al4V material are discussed using the proposed fatigue damage model: first, the changing trend of fatigue life with the ratio $R_{E}$ under different stress amplitudes; second, the changing law of fatigue life along with the ratio $R_{E}$ under different stress ratios.

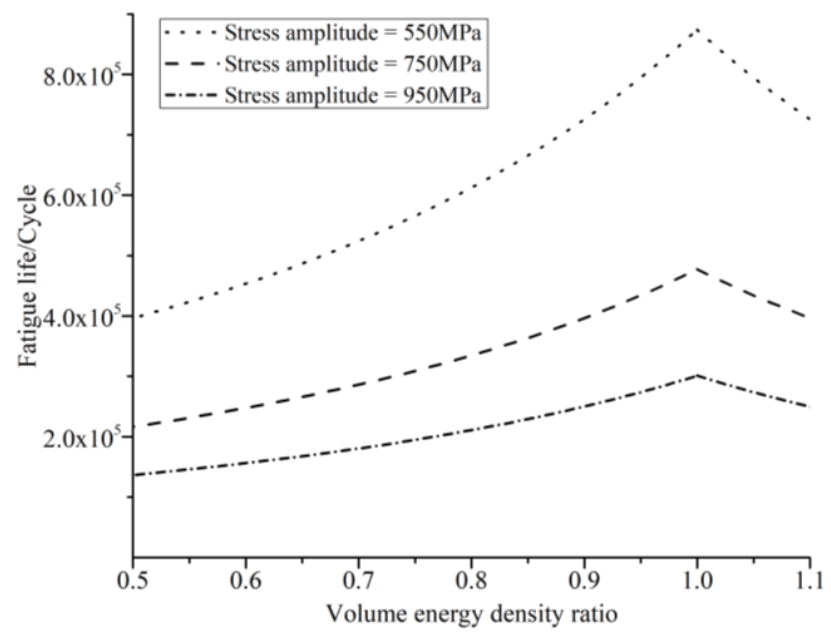

Fig. 5 Variation curve of fatigue life with volume energy density ratio under different stress amplitudes for AM Ti6Al4V

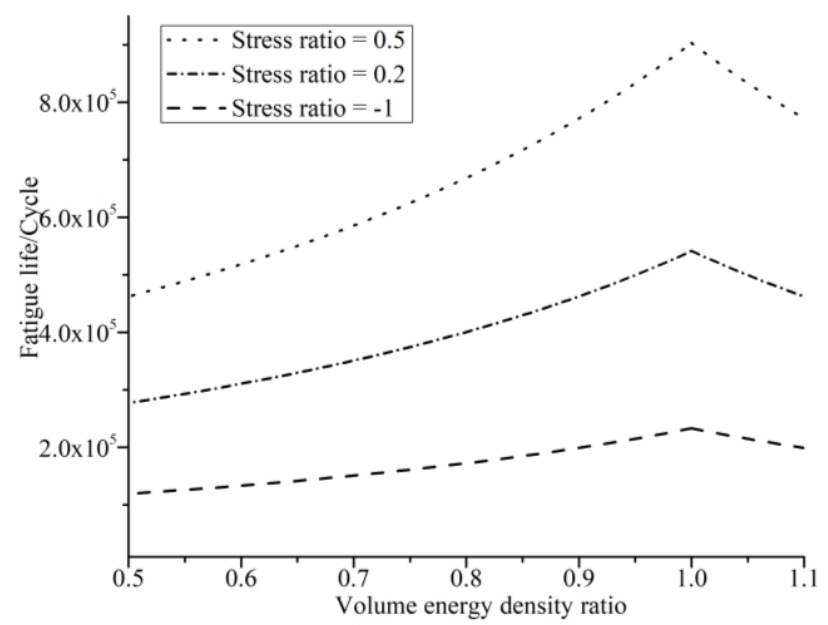

Fig. 6 Variation curve of fatigue life with volume energy density ratio under different stress ratios for AM Ti6Al4V

The changing curve of fatigue life with volume energy density ratio $R_{E}$ at different stress amplitudes is shown in Fig. 5. First, it is seen that the fatigue life increases with the increase of $R_{E}$ if the volumetric energy density ratio is smaller than 1.0. Second, when the ratio $R_{E}$ is less than 1.0, the smaller the stress amplitude is, the more quickly the growth trend of fatigue life is. The larger the stress amplitude is, the less obvious the increasing trend of fatigue life is. Third, if $R_{E}$ is greater than 1.0, the fatigue life reduces along with the increase of the ratio $R_{E}$. Fig. 6 is shown for the changing curve of fatigue life with volume energy density ratio $R_{E}$ at different stress ratios. First, it is clear that the fatigue life increases rapidly with the increase of $R_{E}$ when $R_{E}$ is smaller than 1 . Second, for the same volume energy density ratio, the greater the stress ratio is, the longer the fatigue life is. Third, for different stress ratios, the fatigue life of AM Ti6Al4V reaches the maximum value when $R_{E}$ reaches 1.0 .

\section{Conclusions}

In our work, a fatigue damage model considering AM effects is presented to calculate the fatigue life of AM materials, and the theoretical model and numerical method are verified by the experimental data. Furthermore, the fatigue dam-age characteristics of AM SS316L and Ti6Al4V materials are studied in detail by the new fatigue damage model, which is proved to be a feasible approach for predicting the fatigue life of AM metal materials in engineering. Some of the main results are summarized below:

1. Based on the CDM theory, the elastoplastic constitutive model with damage and the fatigue damage evolution equation considering AM effects are presented. The calibration method of material parameters is presented. Furthermore, the numerical method of the theoretical model is implemented.

2. The fatigue lives of AM SS316L and Ti6Al4V metal materials are predicted and compared with the test results to verify the applicability of the theoretical model.

3. The ratio $R_{\mathrm{E}}$ determines the fatigue life and the fatigue damage evolution characteristics. When $R_{\mathrm{E}}$ is less than 1.0, the fatigue life increases rapidly with the increase of $\mathrm{RE}$. At the same ratio $R_{\mathrm{E}}$, the greater the stress ratio is, the longer the fatigue life is.

\section{References}

1. Guo, N.; Leu, M. C. 2013. Additive manufacturing: technology, applications and research needs, Frontiers of Mechanical Engineering 8(3): 215-243.

2. Goh, G.D.; Yap, Y.L.; Agarwala, S; Wai Y.Y. 2019. Recent progress in additive manufacturing of fiber reinforced polymer composite, Advanced Materials Technologies 4, 1: 1800271.

3. Zhan, Z. X. 2019. Experiments and numerical simulations for the fatigue behavior of a novel TA2-TA15 titanium alloy fabricated by laser melting deposition, International Journal of Fatigue 121: 20-29.

4. Zhang, M; Sun, C. N.; Zhang, X; Goh, P. C.; Wei, J., Hardacre, D., Li, H. 2017. Fatigue and fracture behaviour of laser powder bed fusion stainless steel 316L: Influence of processing parameters, Materials Science and Engineering: A 703: 251-261.

5. Zhang, M.; Sun, C. N.; Zhang, X.; Goh, P. C.; Wei, J.; Li, H.; Hardacre, D. 2018. Elucidating the relations between monotonic and fatigue properties of laser powder bed fusion stainless steel 316L, JOM 70(3): 390-395.

6. Zhang, M.; Sun, C. N.; Zhang, X.; Wei, J.; Hardacre, D.; Li, H. 2018. Predictive models for fatigue property of laser powder bed fusion stainless steel 316L, Materials \& Design 145: 42-54.

7. Carneiro, L.; Jalalahmadi, B.; Ashtekar, A.; Jiang, Y. 2019. Cyclic deformation and fatigue behavior of additively manufactured 17-4 PH stainless steel, International Journal of Fatigue 123: 22-30. 
8. Bagehorn, S.; Wehr, J.; Maier, H. J. 2017. Application of mechanical surface finishing processes for roughness reduction and fatigue improvement of additively manufactured Ti-6Al-4V parts, International Journal of Fatigue 102: 135-142.

9. Pegues, J.; Roach, M.; Williamson, R. S.; Shamsaei, N. 2018. Surface roughness effects on the fatigue strength of additively manufactured Ti-6Al-4V, International Journal of Fatigue 116: 543-552.

10. Sheridan, L.; Gockel, J. E.; Scott-Emuakpor, O. E. 2019. Primary processing parameters, porosity production, and fatigue prediction for additively manufactured alloy 718, Journal of Materials Engineering and Performance 28, 9: 5387-5397.

11. Zhan, Z. X.; Li, H.; Lam K. Y. 2019. Development of a novel fatigue damage model with AM effects for life prediction of commonly-used alloys in aerospace, International Journal of Mechanical Sciences 155: 110-124.

12. Rege, K.; Pavlou, D.G. 2017. A one-parameter nonlinear fatigue damage accumulation model, International Journal of Fatigue 98: 234-246.

13. Zhan, Z. X.; Hu, W. P.; Meng, Q. C.; Shi, S. D. 2016. Continuum damage mechanics-based approach to the fatigue life prediction for 7050-T7451 aluminum alloy with impact pit, International Journal of Damage Mechanics 25, 7: 943-966.

14. Lin Si-Jian, Long Wei, Tian Da-Qing, Liao Jun-Bi. 2018. A new fatigue damage accumulation model considering loading history and loading sequence based on damage equivalence, International Journal of Damage Mechanics 27, 5: 707-728.

15. Zhan, Z. X; Meng, Q. C.; Hu, W. P.; Sun, Y.; Shen, F.; Zhang, Y. J. 2017. Continuum damage mechanics based approach to study the effects of the scarf angle, surface friction and clamping force over the fatigue life of scarf bolted joints, International Journal of Fatigue 102: 59-78.

16. Zhan, Z. X.; Li, H. 2021. Machine learning based fatigue life prediction with effects of additive manufacturing process parameters for printed SS $316 \mathrm{~L}$, International Journal of Fatigue 142: 105941.

17. Huang, J. W.; Meng, Q. C.; Zhan, Z. X.; Hu, W. P.; Shen, F. 2019. Damage mechanics-based approach to studying effects of overload on fatigue life of notched specimens. International Journal of Damage Mechanics 28(4): 538-565.

18. Zhan, Z. X.; Hu, W. P.; Zhang, M.; Meng, Q. C. 2015. The fatigue life prediction for structure with surface scratch considering cutting residual stress, initial plasticity damage and fatigue damage, International Journal of Fatigue 74: 173-182.

19. Zhan, Z. X.; Hu, W. P.; Li, B. K.; Zhang, Y. J.; Meng, Q. C.; Guan, Z. D. 2017. Continuum damage mechanics combined with the extended finite element method for the total life prediction of a metallic component; International Journal of Mechanical Sciences 124: 48-58.

20. Lemaitre, J.; Desmorat, R. 2005. Engineering damage mechanics: ductile, creep, fatigue and brittle failures. Springer.
21. Chaudonneret, M. 1993. A simple and efficient multiaxial fatigue damage model for engineering applications of macro-crack initiation, J Eng Mater - Trans ASME 115(4): 373-379.

22. Zhan, Z. X.; Hu, W. P.; Shen, F; Meng, Q. C.; Pu, J.; Guan, Z. D. 2017. Fatigue life calculation for a specimen with an impact pit considering impact damage, residual stress relaxation and elastic-plastic fatigue damage, International Journal of Fatigue 96: 208-223.

23. Zhan, Z. X. 2018. Fatigue life calculation for TC4TC11 titanium alloy specimens fabricated by laser melting deposition, Theoretical and Applied Fracture Mechanics 96: 114-122.

24. Li, P.; Warner, D. H.; Fatemi, A.; Phan, N. 2016. Critical assessment of the fatigue performance of additively manufactured $\mathrm{Ti}-6 \mathrm{Al}-4 \mathrm{~V}$ and perspective for future research, International Journal of Fatigue 85: 130-43.

25. Liu, Q. C.; Elambasseril, J.; Sun, S. J.; Leary, M.; Brandt, M.; Sharp, P. K. 2014. The effect of manufacturing defects on the fatigue behaviour of Ti-6Al-4V specimens fabricated using selective laser melting, Advanced Materials Research 891: 1519-24.

26. Van, H. B.; Boonen, R.; Moens, D.; Kruth, J. P.; Sas, P. 2012. On the determination of fatigue properties of Ti6Al4V produced by selective laser melting, 53 ${ }^{\text {rd }}$ AIAA/ASME/ASCE/AHS/ASC Conference. 1733.

\section{H. Hong, J. Wang, L. Peng}

\section{A REVISED APPROACH FOR THE LIFE PREDICTION OF METAL MATERIALS FABRICATED BY ADDITIVE MANUFACTURING}

S u m m a r y

In this work, a new fatigue damage model considering the additive manufacturing (AM) effects is established. We first present the elastoplastic constitutive model with the newly established fatigue damage model considering AM effects. The method to calibrate the material parameters is put forward, and the numerical solution of the theoretical model is implemented. Second, the fatigue lives of two AM metal materials are predicted, and the applicability of the theoretical model is verified by the test results. Finally, the influence of the volume energy density ratio on the fatigue life of AM metal materials is analyzed, and the results show that the volume energy density ratio has a great influence on the fatigue behavior of AM metal materials. When the ratio is less than 1.0, the fatigue life increases rapidly with the ratio increases. The fatigue life becomes longer with the stress ratio when the volume density ratio keeps as a constant. The research work in this paper provides a feasible meth-od to predict the fatigue life of AM metal materials by continuum damage mechanics in engineering.

Keywords: numerical simulation; life prediction; additive manufacturing; fatigue model; volume energy density.

Received December 26, 2020 Accepted June 02, 2021 